\title{
Memory scanning of described images and undescribed images: Hemispheric differences
}

\author{
CHRIS T. BERSTED \\ Southwest Missouri State University, Springfield, Missouri 65802
}

\begin{abstract}
Seamon (1972) has found that reaction times (RTs) to memory probes do not increase with memory set size (M) for words encoded in mental images in a memory scanning task. Rothstein and Atkinson (1975) have failed to replicate Seamon's results. Experiment 1 investigated this discrepancy by manipulating one methodological difference (whether images were or were not described) between the two investigations. Results revealed that described images produced typical linear increases in RT with M. Undescribed images, however, revealed no change in RT with $\mathbf{M}$ for positive probes (i.e., the word presented was contained in the current memory set). Experiment 2 manipulated to which visual field probes were presented (RVF = right visual field; LVF = left visual field). Results showed that the imagery group showed no relation between RT and $M$ for probes presented to the LVF, but a linear relation for probes presented to the RVF. Described-image and repetition groups revealed a linear relation between RT and $M$ no matter which visual field received the probe.
\end{abstract}

Since Sternberg (1966) introduced the memory scanning task, hundreds of studies have been performed to investigate the type of processing that occurs in item recognition. In this paradigm, a small number of stimuli (the memory set) is presented to the subject. A probe or test stimulus is then presented to the subject. The subject's task is to make a "yes" response if the probe is a member of the memory set and a "no" response if it is not. Sternberg (1966) found a linear function for the relationship between reaction time (RT) and memory set size (M). He concluded that subjects engaged in a serial search process in which subjects sequentially com. pared the encoded test stimulus against the memorial representations of each of the items in the memory set. In addition, he concluded that the serial search process was exhaustive because the slopes of "yes" and "no" RTs in relation to memory set were parallel. In general, a monotonic increase in RT with $M$ has typically been found (e.g., J. A. Anderson, 1973; Briggs, 1974; Nickerson, 1972).

Seamon (1972) has presented an interesting exception to the generalization that RT is linearly related to $M$. He found that a group of subjects instructed to use relational imagery to remember the words in each memory set (varying in size from one to three words) showed no increase in RT with M. A second group in Seamon's investigation using a repetition strategy to remember words in the memory sets exhibited the more common linear increase of RT with M. Seamon suggested that memory codes for repetitively rehearsed memory sets required a sequential comparison process, whereas words represented in a visually imagined scene could be processed in parallel. ${ }^{1}$ Similarly, Paivio (1971) has suggested that imagery leads to a visual memory code that he believes is a parallel processing system, in contrast to verbal processing, which is thought to operate in a sequential manner.

Rothstein and Atkinson (1975), however, attempted and failed to replicate Seamon's (1972) results. They found approximately equal linear increases in RT with $M$ for both an imagery and a repetition group. They did note, however, a lesser slope for "no" responses than for "yes" responses, regardless of instructional set.

A second investigation centered on Seamon's (1972) data confuses the issue further. Kerst (1976) (among other conditions) added a group asked to form a sentence with the words from each memory set. He reasoned that Seamon's findings may have been due to an interaction of unspecified modality rather than interacting mental images. Thus, if a sentence-generation condition involved serial comparisons and an imagery condition involved parallel comparisons, then one might argue that these two conditions generate "different kinds of retrieval (and perhaps storage) processes" (Kerst, 1976, p. 574). His findings were that the repetition and sentence instructional groups produced the typical linear increase in RT with $M$. For the imagery group, however, when "yes" and "no" responses were analyzed separately, only the "no" responses produced a linear increase in RT with M. Kerst argues that since both "yes" and "no" slopes should show a lack of a linear relation between $\mathrm{RT}$ and $\mathrm{M}$, his results were not supportive of Seamon's parallel scanning conclusion.

An attempt at a synthesis of these disparate results is difficult. However, if one only considers "yes" responses, the evidence from Kerst (1976) and Seamon (1972) 
suggest the possibility of processing objects (representing words) within a single image in parallel. Rothstein and Atkinson's (1975) data, on the other hand, are inconsistent with this possibility. The Kerst investigation gives rise to a possible reason why Rothstein and Atkinson's attempted replication may have failed. Kerst found that instructions to produce sentences containing words in memory sets produced a linear relation between $M$ and RT. Prior to their testing for each memory set (and unlike Seamon's original study), Rothstein and Atkinson asked their subjects to describe their images aloud. This description (presumably sentence-like) might possibly have led to a separate or at least elaborated (see J. T. Anderson \& Bower, 1973) memory code, or a greater depth or breadth of encoding (Craik \& Lockhart, 1972), not normally available to subjects using an imagery strategy to remember words in a memory set. In fact, some support exists for this suggestion of a separate or elaborated memory code due to the conjunction of imagery and verbal description. For instance, Bahrick and Boucher (1968) demonstrated that encouraging verbalization (i.e., description) of pictures during presentation enhanced subsequent recall of pictures. In addition, Bersted (Note 1) has found that subjects asked to describe visual images used to remember paired associates showed recall superior to that of subjects not describing their images. Thus, it seems at least possible that subjects asked to describe their images have a different kind of memory, or memory search strategy, than subjects not asked to describe their images.

\section{EXPERIMENT 1}

In order to investigate the possibility that describing one's image changes search strategy, the present research compared two groups of subjects given imagery instructions to remember memory sets, using a procedure virtually identical to that of Rothstein and Atkinson (1975). The groups differed only in that one group was asked to describe their images prior to testing. In addition, unlike previous investigations, memory sets of Size 4 were also tested.

\section{Method}

Subjects. The subjects were 16 right-handed undergraduates recruited from an introductory psychology course as an optional means of fulfilling a class requirement. The subjects were randomly assigned to imagery and imagery + description groups, with the constraint that sex was balanced.

Apparatus. An Iconix three-channel projection tachistoscope was used for presentation of stimuli on a back-lighted projection screen. A Gerbrands millisecond clock was used for measuring RT. Three telegraph keys, each $2.5 \mathrm{~cm}$ in diameter, were mounted in a semicircle on a board in front of the subjects and were separated by $1.0 \mathrm{~cm}$.

Stimuli. The stimuli were 96 nouns selected from the Paivio, Yuille, and Madigan (1968) norms. These nouns all had I values of 6.2 or greater, based on the above norms, and varied in length from three to eight letters. These stimuli were photographed and reproduced as slides (black letters on a white background). Each word subtended approximately 1.5-2.5 deg of retinal arc in a horizontal direction when projected on the screen. For memory set slides (varying from one to four words), the words when projected were relatively smaller (.7-1.5 deg of retinal arc).

Procedure. Memory sets consisted of one, two, three, or four words. These words were randomly selected without replacement to form memory sets and negative probes. Each memory set was used for a block of eight consecutive trials, with four trials using positive probes (i.e., a single word drawn from the current memory set), and four trials using a negative probe (i.e., a word not experienced before).

Two sets of 112 trials were employed in the experiment, with different randomly selected memory sets. Each set was administered to half the subjects in each memory condition. The first 16 trials, with two positive and two negative probes for each of the Ms one through four, were designated as practice trials. These 16 trials were followed by 96 trials, consisting of three 8-trial blocks for each M. Within each of the two sets of 96 test trials, half the subjects in each memory condition received one ordering of the blocks of eight trials and the other half received a second ordering of the blocks, with the constraint that each consecutive set of four eight-trial blocks contained one block of each of the four Ms.

Memory sets for each block were presented prior to each block of trials on the back-lighted projection screen. Prior to presentation of the first memory set, subjects read instructions for the experiment. Subjects in the imagery group were told to visualize (imagine) a scene that contained each of the objects corresponding to the words in the memory set and to say "OK" when they had the image in mind. Subjects in the imagery + description group were given the same instructions, with the exception that when a new memory set was presented, they were asked to describe aloud their image rather than say "OK." Following the subjects' verbal response, an audible click occurred. A fixation point (small circle of light) appeared on the screen $2.5 \mathrm{sec}$ after the click. A probe word appeared on the screen $.5 \mathrm{sec}$ later, centered from left to right where the fixation point had been; it remained on until the subject responded. The subject was told to rest his right index finger on the center telegraph key at the start of each trial and to respond "yes" (i.e., the probe was a member of the current memory set) by as quickly as possible pressing the key to his right and "no" by as quickly as possible pressing the key to his left. RT was measured from the onset of the probe stimulus until a key was pressed by the subject. After each trial within a memory set block, all subjects were instructed that prior to the next trial they should refresh their image and say "OK" when they had their image clearly in mind. Each experimental session took approximately $30 \mathrm{~min}$. Thus, the only difference between the two groups of subjects was that the imagery + description group had to describe their image once prior to the first test trial for each memory set.

\section{Results}

Initial analyses indicated that the two sets of trials and the order factor within sets were not significant variables, and all remaining analyses were collapsed across these variables. Error rates for both memory strategy conditions were less than $1 \%$, and all trials on which erroneous responses occurred were repeated within the same block of trials.

The first analysis was a 2 (strategy: imagery vs. imagery + description) by $4(M=1,2,3,4)$ by 2 (response type: "yes" vs. "no") ANOVA using the average (from the three blocks of trials for particular M) median (over the four probes) RT for each subject. Four significant 
effects were found. First, the $M$ variable was significant $[\mathrm{F}(3,42)=33.70, \mathrm{p}<.01, \mathrm{MSe}=.025]$. Reaction times for $M=1.4$ were $.591, .608, .664$, and $.650 \mathrm{sec}$, respectively. Second, the response type variable was significant $[F(1,14)=16.59, \quad p<.01, \quad M S e=.199]$. "Yes" responses $(.584 \mathrm{sec})$ were faster than "no" responses $(.662 \mathrm{sec})$.

Third, there was a significant interaction between memory strategy and $\mathrm{M}[\mathrm{F}(3,42)=7.54, \mathrm{p}<.01, \mathrm{MSe}=$ $.007]$. This interaction resulted from a smaller slope relating $\mathrm{RT}$ with $\mathrm{M}$ for the imagery vs. the imagery + description group. This interaction, however, was qualified by a significant three-factor interaction among memory strategy, $M$, and response type $[F(3,42)=11.26$, $\mathrm{p}<.01, \mathrm{MSe}=.007]$. Figure 1 depicts this interaction. It can be noted that for "yes" responses (positive probes), the slope relating RT and $\mathrm{M}$ is less for the imagery than for the imagery + description group. For "no" responses, no apparent difference in slopes exists. Included in Figure 1 are the equations for the bestfitting regression lines for the imagery and imagery + description groups for both "yes" and "no" response trials.

In order to further investigate the three-factor interaction reported above, simple effects were investigated with slopes based on average median RTs for individual subjects as the dependent variable. This analysis revealed that the slope relating $M$ to $R T$ was indeed smaller for the imagery than for the imagery + description group for "yes" responses $[F(1,28)=42.49, p<.01]$ and that the slopes for both the imagery $[F(1,14)=18.72, p<.01]$ and the imagery + description $[F(1,14)=12.94, p<.01]$ groups were different on "yes" and "no" response trials.

A similar analysis, but using intercept values for the best-fitting regression lines of individual subjects, revealed a larger intercept value for the imagery group compared to the imagery + description group for both "yes" $[F(1,28)=111.07, p<.01]$ and "no" $[F(1,28)=$ $20.42, p<.01]$ responses. Finally, the intercept value was less on "yes" than on "no" responses for the imagery + description group $[F(1,14)=24.51, p<.01]$.

\section{Discussion}

The results from the present research clarify an apparent conflict in past results concerning images in a memory scanning task. The findings of a nonsignificant slope relating $\mathrm{RT}$ and $\mathrm{M}$ for the imagery group on positive trials is consistent with and extends to Set Size 4 the results of both Kerst (1976) and Seamon (1972). In addition, the finding of a significant relation between RT and $M$ on both negative and positive trials for the imagery + description group suggests that Rothstein and Atkinson's (1975) apparent failure to replicate was probably due to their methodological change requiring subjects to describe their images. Thus, at least for positive trials, support is given to a parallel scanning hypothesis (or any other conceptualization that would

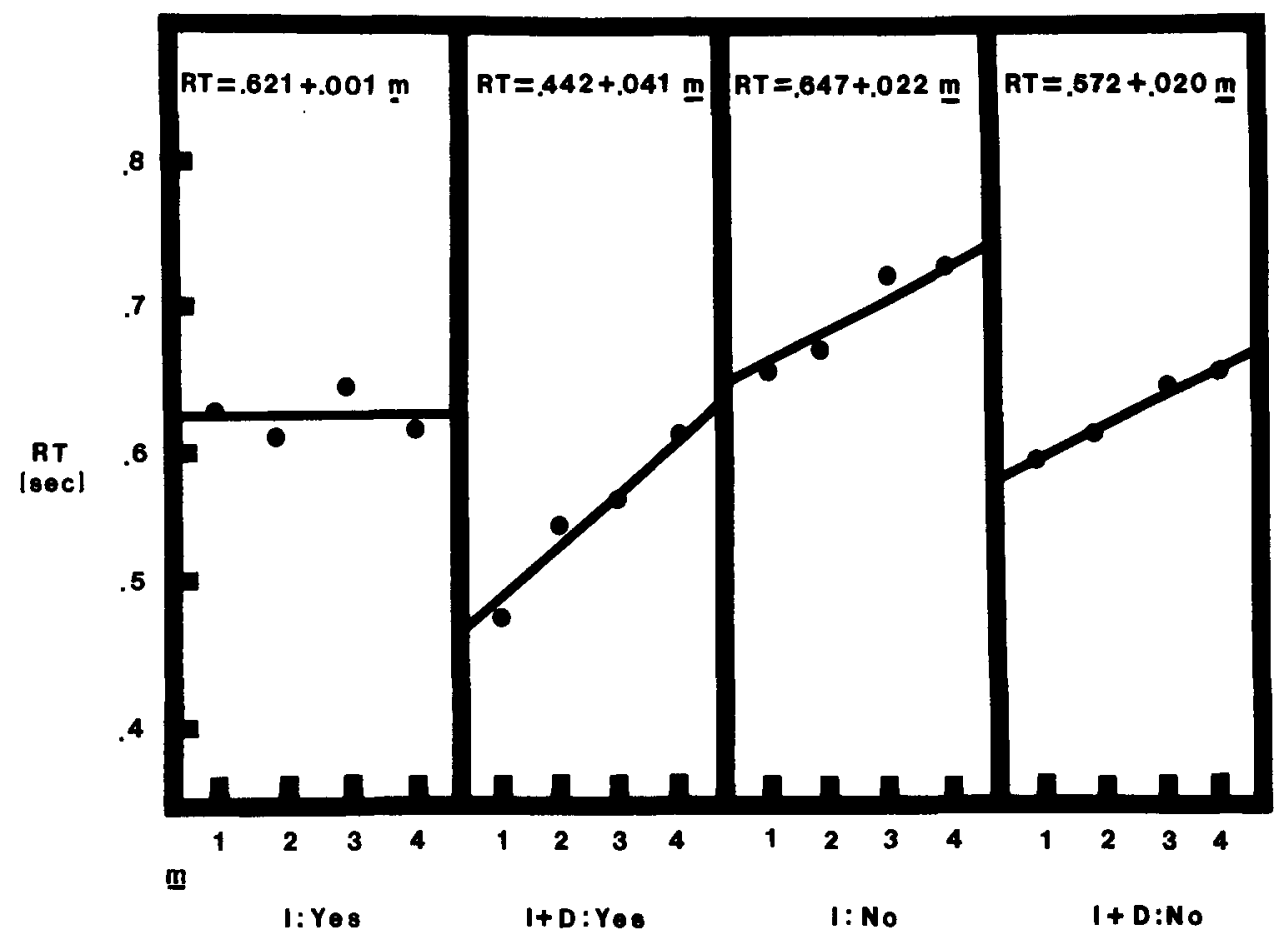

Figure 1. Average median reaction time (RT) in seconds on positive (yes) and negative (no) probes as a function of memory instructions and memory set size (M). 
predict response times independent of the size of the memory load) for undescribed images.

The question remains, however, as to how describing one's image apparently leads to the processing differences found on positive probes. A possible speculative explanation involves hemispheric differences revealed by perceptual and/or cognitive asymmetries. For example, Kimura (1973) has suggested a direct-access model of hemispheric specialization. First, it was suggested that the left cerebral hemisphere was specialized for verbal processing and the right cerebral hemisphere was specialized for visuospatial processing. Second, it was predicted that performance would be better (accuracy and/or time) for a direct pathway to the appropriately specialized hemisphere than for an indirect pathway necessitating transcallosal information transfer, and perhaps degradation or loss of information.

One consequence of the above line of reasoning was a study by Seamon and Gazzaniga (1973), later replicated by Metzger and Antes (1976, Experiment 2). One group used a repetition strategy (repeat the words of a memory set), and a second group used an imagery strategy (form an image that contains the objects that represent the pair of words) to remember each pair of words. Using RT as the dependent variable, they found a left visual field (LVF)/right hemisphere (RH) advantage (i.e., faster RT) for picture probes selectively input to the hemispheres using an imagery strategy and a right visual field (RVF)/left hemisphere (LH) advantage for the repetition group. Bersted (Note 2) has replicated this finding using word probes and has also showed that the use of a sentence to remember the pair of words produced an RVF-LH advantage similar to Seamon and Gazzaniga's repetition group. Thus, perhaps the processing differences for positive probes found in Experiment 1 are related to hemisphere specialization.

More recently, two modifications to this direct model have been suggested. First, the differences between the hemispheres may be more a matter of degree than a strict dichotomy. Thus, either hemisphere may be able to adequately process at least simple verbal or visual information, but their efficiencies might vary. Second, rather than a simple verbal-visuospatial dichotomy between the hemispheres, differences in processing strategies also exist (e.g., Helige, 1980; Madden \& Nebes, 1980; Moscovitch, 1979). The LH is characterized as processing information in an abstract, analytic (serial) manner, whereas the $\mathrm{RH}$ is characterized as processing information in a holistic, perceptual (parallel) manner. Indeed, it has been argued by Helige (1980, p. 650) that "qualitatively different processing techniques may vary from trial to trial and be introduced as a result of hemisphere of presentation." If Helige is correct, then different relations between RT and M might be found based on the hemisphere of probe presentation, and in fact, he has presented some data consistent with this prediction. In addition, if Paivio (1971) is correct in his dual code of imagery formulation (imagery produces both a verbal and a visual memory code), then one might expect parallel processing of images if the probe is presented to the $\mathrm{RH}$ and serial processing if the probe is presented to the LH. Probably the same expectations would hold for described images. Thus, either memory strategy could produce data consistent with parallel or serial scanning, depending on which hemisphere received the probe. The difference found in Experiment 1, then, would presumably be due to a biasing of which hemisphere would be involved in processing when probes are presented to both hemispheres simultaneously.

\section{EXPERIMENT 2}

Experiment 2 combined lateralized presentation of probes (either to the RVF-LH or to the LVF-RH) for varied Ms. It was felt that the combination of these two methodologies would provide evidence whether the differential relationships found between $R T$ and $M$ as a function of memory strategy in Experiment 1 could be the result of selective hemispheric involvement. If in fact the two hemispheres of the brain can or typically do use different processing strategies (analytic-serial vs. holisticparallel), then different functions (an increase vs. no increase in RT as a function of $M$ ) might be found for a single memory strategy depending upon which hemisphere received the probe.

\section{Method}

Subjects. Thirty right-handed undergraduates recruited from the same pool of subjects as Experiment 1 were randomly assigned to imagery, imagery + description, and repetition groups, with the constraint that sex was balanced.

Apparatus. The apparatus used was the same as in Experiment 1 .

Stimuli. The stimuli were 66 nouns selected and reproduced in a fashion similar to Experiment 1.

Procedure. Memory sets consisted of one, two, or three words. These words were randomly selected without replacement to form both memory sets and negative probes. Each memory set was used for a block of eight consecutive trials, with four trials using positive probes (i.e., a single word drawn from the current memory set) and four trials using a negative probe (i.e., a word not presented previously).

Two sets of 84 trials were employed in the experiment, with different randomly selected memory sets and probes. Each set was administered to half the subjects in each memory condition. The first 12 trials, with two positive and two negative probes for each of the Ms one through three, were designated as practice trials. These 12 trials were followed by 72 trials, consisting of three 8-trial blocks for each $\mathrm{M}$.

Memory sets for each block were presented prior to each block of trials on the back-lighted projection screen. Prior to the presentation of the first memory set, subjects read instructions for the experiment. Subjects in the imagery and the imagery + description groups were given instructions similar to those in Experiment 1. Subjects in the repetition group were asked to repeat aloud twice the words in the memory set. Following the subjects' verbal response ("OK," description, or repetition) a click occurred. A fixation point (small circle of light) appeared on the screen 2 sec after the click. A probe word appeared on 
the screen $.5 \mathrm{sec}$ later. This probe word was located either to the left or to the right of the fixation point by approximately $2 \mathrm{deg}$ of retinal arc. (If the probe word was to the subjects' right of the fixation point, then the first letter of the word was $2 \mathrm{deg}$ of arc from the fixation point, whereas when the probe occurred to the left of the fixation point, the last letter of the probe was $2 \mathrm{deg}$ of arc from the fixation point.) For each memory set, half of the positive probes (the word presented was a member of the current memory set) and half of the negative probes (the word presented was not a member of the current memory set) were presented to each visual field. Each probe word was projected on the screen for $150 \mathrm{msec}$.

RT was measured in the same manner as in Experiment 1. After each trial within a memory set block, subjects within the imagery and imagery + description groups were instructed to refresh their image and say "OK" when they had their image clearly in mind. Subjects in the repetition group were instructed to again repeat aloud twice the words in their current memory set. Each experimental session took approximately $30 \mathrm{~min}$.

\section{Results}

Initial analyses indicated that the two sets of trials were not a significant variable, and all remaining analyses were collapsed across this variable. Error rates for all memory strategy conditions were less than $1 \%$, and all trials on which erroneous responses occurred were repeated within the same block of trials. Since Experiment 1 had demonstrated that a lack of a relation between RT and M might be expected only on "yes" trials (positive probe), "yes" and "no" trials were analyzed separately.

The first analysis was a 3 (strategy: imagery, imagery + description, and repetition) by $3(M=1,2$, or 3$)$, by 2 (visual field: RVF vs. LVF) ANOVA using the average (from the three blocks of trials for a particular M) median (over the four probes) RT for each subject for positive probes. Two main effects and four interactions were found to be significant. First, the $M$ variable was significant $[F(2,54)=79.27, p<.01, \mathrm{MSe}=.001]$. Mean RTs for $M=1-3$ were 648,690 , and $722 \mathrm{msec}$, respectively. The second main effect was due to the hemisphere variable $[F(1,27)=5.81, p<.05, M S e=.008]$. The mean RT for the RVF was $671 \mathrm{msec}$, and for the LVF, it was $702 \mathrm{msec}$.

The interaction between memory strategy and $M$ was significant $[\mathrm{F}(4,54)=3.03, \mathrm{p}<.05, \mathrm{MSe}=.001]$. This interaction was produced by a smaller increase in RT as a function of $M$ for the imagery than the other two memory strategy groups (for $M=1-3$ : imagery = 703,730 , and $748 \mathrm{msec}$; imagery + description $=667$, 715 , and $759 \mathrm{msec}$; repetition $=575,624$, and $660 \mathrm{msec}$, respectively).

The interaction between $M$ and visual field was also significant $[F(2,54)=4.94, p<.05, \mathrm{MSe}=.001]$. This interaction resulted from a smaller increase in $\mathrm{RT}$ as a function of $\mathrm{M}$ for the LVF compared to the RVF (for $M=1-3: L V F=675,704$, and $729 \mathrm{msec} ; R V F=622$, 675 , and $715 \mathrm{msec}$, respectively).

In addition, the Memory Strategy by Visual Field interaction was significant $[F(2,27)=6.00, p<.01$, $\mathrm{MSe}=.008]$. This interaction resulted from an LVF superiority for the imagery group (LVF $=711, \mathrm{RVF}=$ $743 \mathrm{msec}$ ), but an RVF superiority for both the described imagery (LVF $=745, \mathrm{RVF}=682 \mathrm{msec}$ ) and the repetition (LVF $=651, \mathrm{RVF}=587 \mathrm{msec}$ ) groups.

All of the above interactions were qualified, however, by the interaction among memory strategy, $\mathrm{M}$, and visual field $[F(4,54)=2.80, p<.05$, MSe $=.001]$. Figure 2 depicts this interaction. As can be noted, all memory strategies exhibited approximately linear functions relating $\mathrm{RT}$ to $\mathbf{M}$ when probes were presented to

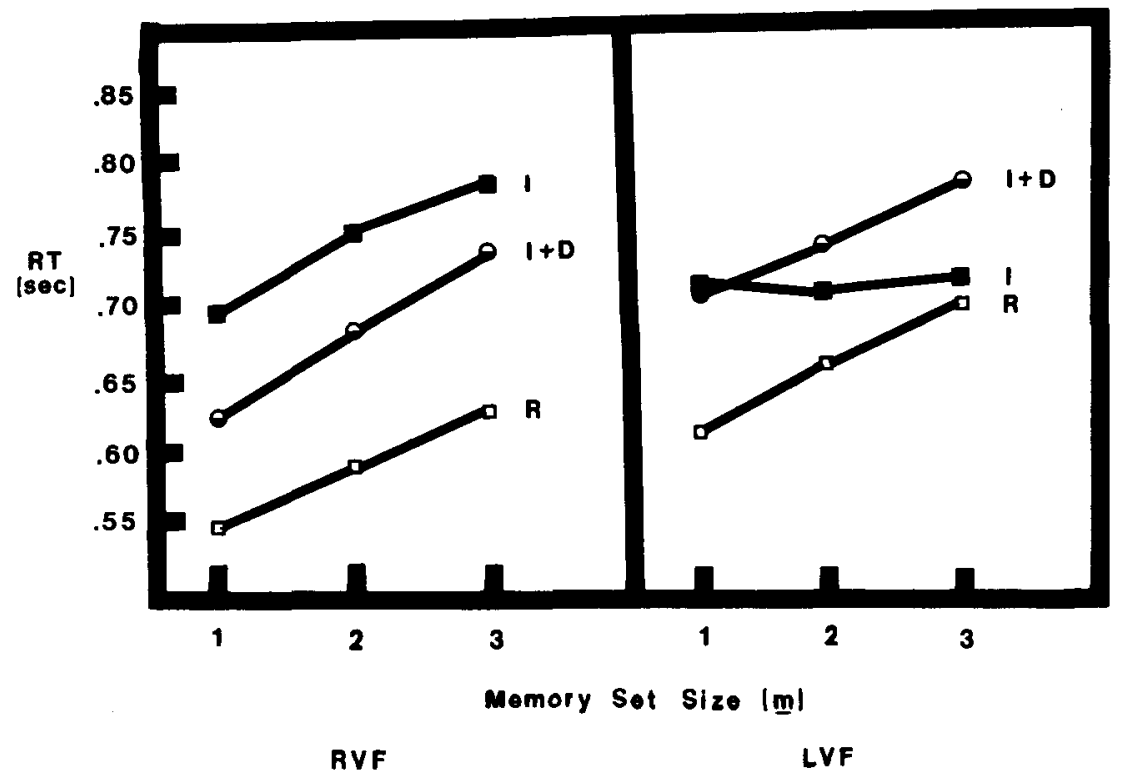

Figure 2. Average median reaction time (RT) in seconds for positive probes as a function of memory instruction ( $=$ imagery; $I+D=$ described image; $R=$ repetition), memory set size $(M)$, and the visual field the probe was presented to $(R V F=$ right visual field ; LVF $=$ left visual field). 
Table 1

Parameters for Regression Lines (in Milliseconds) for Memory Strategy, Hemisphere, and Response Type

\begin{tabular}{lccccc} 
& & \multicolumn{3}{c}{ Hemisphere } \\
\cline { 3 - 5 } Memory & Response & \multicolumn{2}{c}{ Left } & & \multicolumn{2}{c}{ Right } \\
\cline { 3 - 5 } \cline { 5 - 6 } Strategy & Type & Intercept & Slope & Intercept Slope \\
\hline \multirow{2}{*}{ Imagery } & Yes & 655 & 45 & 708 & 1 \\
& No & 707 & 17 & 723 & 22 \\
Imagery + & Yes & 566 & 56 & 674 & 35 \\
Description & No & 646 & 16 & 702 & 14 \\
Repetition & Yes & 508 & 40 & 560 & 46 \\
& No & 624 & 21 & 626 & 23 \\
\hline
\end{tabular}

the RVF, but only the imagery group resulted in no apparent relationship between RT and $\mathrm{M}$ when probes were presented to the LVF. Regression analysis for the memory strategies separated by visual field revealed a slope of $1 \mathrm{msec}$ for the imagery group in the LVF, whereas all other conditions had an average slope of $44 \mathrm{msec}$, with a range of $35.56 \mathrm{msec}$. Table 1 shows the parameters found for these regression lines.

A similar analysis was also conducted for the negative probes ("no" responses). Only the $M$ variable was significant $[F(2,54)=13.40, p<.01, \quad M S e=.019]$. This resulted from an increase in RT as a function of $\mathrm{M}$ (mean RTs for $M=1-3$ were 687,708 , and $723 \mathrm{msec}$, respectively). The hemisphere variable seemed to indicate that probes directed to the RVF produced faster RTs $(.695 \mathrm{sec})$ than probes to the LVF $(.716 \mathrm{sec})$, although this effect was only marginally significant $[F(1,27)=4.19, p<.053, \mathrm{MSe}=.023]$. Table 1 also includes parameters for regression lines found for negative probes.

\section{Discussion}

The results for positive probes from Experiment 2 for the imagery group are consistent with the contention that the relation between $R T$ and $M$ (and hence the type of processing) can vary depending on which hemisphere receives the probe (Helige, 1980). This finding also supports the contention that the lack of a relation between RT and $M$ for the imagery group in Experiment 1 may be due to the RH's controlling the comparison process: Comparison of positive probes presented centrally (center of the visual field) for memory sets retained under imagery conditions (Experiment 1) produced results comparable to probes presented to the RH (Experiment 2), but not to probes presented to the $\mathrm{LH}$.

The results for positive probes for the describedimage group, however, are not consistent with the notion that hemisphere of probe presentation determines the type of memory comparison process that will occur. Indeed, the results for the described-image group showed a linear increase in RT with increasing M (like Experiment 1) no matter which hemisphere received the probe. Likewise, the repetition group produced results comparable to the described-image group (i.e., a linear increase in RT with increasing $M$, again irrespective of which hemisphere received the probe).

While the difference found between the imagery and the described-image groups for the RH seems puzzling, it may not be unreasonable to assume that any emphasis upon verbal coding (e.g., describing one's image) may bias subsequent memory comparisons in favor of a more verbal analysis. Kinsbourne's (1970) attentional model of lateralization would be consistent with this notion. He suggested that asymmetries are due to differential hemispheric activation depending on memory load or cognitive set (priming). In this case, the instructions for remembering each memory set would produce differential hemispheric activation due to priming (assuming that the hemispheres are at least partially specialized for verbal or visuospatial processing). Thus, imagery instructions may prime the $\mathrm{RH}$, whereas describing one's image (and repetition instructions) may prime the LH. One would probably have to assume, however, that this priming effect, in some cases at least (e.g., the undescribedimage group), may be only one of a number of factors determining which hemisphere is responsible for memory comparisons. In fact, others (Kirsner, 1980; Sergent, 1982) have suggested that hemispheric superiorities are not due to any one factor. Instead, complex interactions exist between hemispheric processing specializations, stimulus characteristics, task demands, and perhaps other factors as well.

Both Experiment 1 and Experiment 2 found smaller increases in RT on negative than on positive probes for increasing $M$ for all memory conditions (except imagery). Others (Davis \& Schmidt, 1973; Helige, 1980; Moscovitch, 1973) have speculated why functions relating RT and M may differ on "yes" and "no" trials, and this difference will not be discussed here.

\section{GENERAL DISCUSSION}

The results from the two investigations reported here lead to several conclusions. First, parallel processing of images apparently occurs due to $\mathrm{RH}$ involvement. Second, describing one's image leads to qualitatively different memory comparision processes. On the basis of the lateral asymmetry found in Experiment 2 (i.e., faster RT to positive probes directed to the LH than the $\mathrm{RH})$, a reasonable guess is that description of an image somehow biases the comparison process to the $\mathrm{LH}$.

Several cautions, however, should be expressed. First, while the results for the imagery groups in the two experiments are consistent with a serial-parallel distinction between the LH and RH of the brain, respectively, this distinction may be too simplified or inaccurate. For example, Friedman and Polson (1981) have recently argued that the lateral asymmetries may more simply be explained by a "multiple resources framework." 
According to this viewpoint, most tasks require resources (processing) by both hemispheres of the brain. Thus, apparent differences between the hemispheres from this viewpoint would reflect a different mix of the resource utilization from the two hemispheres. Parallel and serial might then be thought of as terms that reflect the differential involvements (rather than one or the other) of the two hemispheres in memory scanning tasks. In addition, parallel (and a similar argument could be made concerning serial) processing is probably more descriptive than explanatory: Other possibilities exist for explaining why no increase in RT might be found with increasing $M$ (see Massaro, 1975, for an example). However, even though parallel and serial might not accurately describe the way in which memory searches and comparisons are conducted, it still should be apparent that different processing strategies exist at least partially dependent on the hemispheres.

Second, the present research, and likewise that of Kerst (1976) and Seamon (1972), does not actually deal with the form of information representation. As J. R. Anderson (1978) and others have argued, behavioral data alone cannot be used to decide between imaginal and propositional representations. Thus, rather than concluding that images (or visual representations, if roughly equivalent) are processed in parallel while other representations are processed in a serial manner, a much more restricted conclusion is warranted. The studies by Coleman and Zenhausern (1979), Helige (1975), Kinsbourne (1975), and Maniscalco and DeRosa (1979), among others, suggest that memory encoding and scanning are highly complex tasks influenced by task demands, type and organization of stimulus materials, and even perhaps physiological differences (e.g., hemispheric dominance) between individuals. Indeed, the fragility of the parallel-serial distinction is exemplified in the present research, in which images produce parallel processing, but only when probes are directed centrally or to the RH.

\section{REFERENCE NOTES}

1. Bersted, C. T. Effects of combining sentence, repetition, and imagery mnemonics for recall of PAs. Unpublished manuscript, 1977.

2. Bersted, C. T. Recognition memory differences to lateralized stimulation following PA learning under imagery, sentence, and rote instructions. Paper presented at the Midwestern Psychological Association, Chicago, May 1976.

\section{REFERENCES}

Anderson, J. A. A theory for the recognition of items from short memorized lists. Psychological Review, 1973, 80, 417-438.

Ande rson, J. R. Arguments concerning representations for mental imagery. Psychological Review, 1978, 85, 249-277.
Anderson, J. T., \& Bower, G. H. Human associative memory. Washington, D.C: Winston, 1973.

Bahnick, H. P., \& Boucher, B. Retention of visual and verbal codes of the same stimuli. Journal of Experimental Psychology, $1968,78,417-422$.

Briggs, G. E. On the predictor variable for choice reaction time. Memory \& Cognition, 1974, 2, 575-580.

Coleman, S., \& Zenhausern, R. Processing speed, laterality patterns, and memory encoding as a function of hemispheric dominance. Bulletin of the Psychonomic Society, 1979, 14, 357-360.

Craik, F. I. M., \& Lockhart, R. S. Levels of processing: A framework for memory research. Journal of Verbal Learning and Verbal Behavior, 1972, 11, 671-684.

Davis, R., \& Schmidt, V. Visual and verbal coding in interhemispheric transfer of information. Acta Psychologica, 1973, 37, 229-240.

DeRosa, D. V., \& Tracz, S. Memory scanning of organized visual material. Journal of Experimental Psychology: Human Learning and Memory, 1976, 2, 688-694.

Friedman, A., \& Polson, M. C. Hemispheres as independent resource systems: Limited-capacity processing and cerebral specialization. Journal of Experimental Psychology: Human Perception and Performance, 1981, 7, 1031-1058.

HeLige, J. Hemispheric processing difference revealed by differential conditioning and reaction time performance. Journal of Experimental Psychology: General, 1975, 104, 309-326.

HeLiGe, J. Effects of perceptual quality and visual field of probe stimulus presentation on memory search for letters. Journal of Experimental Psychology: Human Perception and Performance, $1980,6,639-651$.

KERst, S. M. Interactive visual imagery and memory search for words and pictures. Memory \& Cognition, 1976, 4, 573-580.

Kimura, D. The asymmetry of the human brain. Scientific American, 1973, 33, 193-201.

Kinsbourne, M. The cerebral basis of lateral asymmetries in attention. Acta Psychologica, 1970, 228, 70-78.

KinsBourne, M. The mechanism of hemispheric control of the lateral gradient of attention. In P. M. A. Rabbit \& S. Dornic (Eds.), Attention and performance $V$. New York: Academic Press, 1975.

Kirsner, K. Hemispheric specific processes in letter matching. Journal of Experimental Psychology: Human Perception and Performance, 1980, 6, 167-169.

Madden, D. J., \& Nebes, R. D. Visual perception and memory. In M. C. Wittrock (Ed.), The brain and psychology. New York: Academic Press, 1980.

Maniscalco, C. I., \& DeRosa, D. V. Scanning organized material: Individual differences in search strategies. Bulletin of the Psychonomic Society, 1979, 14, 361-364.

Massaro, D. W. Experimental psychology and information processing. Chicago: Rand-McNally, 1975.

Metzaer, R. L., \& Antes, J. R. Sex and coding strategy effects on reaction time to hemispheric probes. Memory \& Cognition, 1976, 4, 167-171.

Moscovitch, M. Language and the cerebral hemispheres: Reaction time studies and their implications for models of cerebral dominance. In P. Milner, L. Krames, \& T. Alloway (Eds.), Communication and affect: Language and thought. New York: Academic Press, 1973.

Moscovitch, $M$. Information processing and the cerebral hemispheres. In M. S. Gazzaniga (Ed.), The handbook of behavioral neurobiology (Vol. 2). New York: Plenum, 1979.

Nickerson, R. S. Binary-classification reaction time. A review of some studies of human information-processing capabilities. Psychonomic Monograph Supplements, 1972, 4(Whole No. 65), 275-318. 
Paivio, A. Imagery and verbal processes. New York: Holt, Rinehart, \& Winston, 1971.

Paivio, A., Yullle, J. C., \& Madigan, S. A. Concreteness, imagery, and meaningfulness values for 925 nouns. Journal of Experimental Psychology Monograph, 1968, 76(1, Pt. 2).

Rothstein, L. D., \& Atkinson, R. C. Memory scanning for words in visual images. Memory \& Cognition, 1975, 3, 541-544.

Rosenbaum, D. A. Rule use in character classification: Are serial and parallel processing discrete? Memory \& Cognition, 1974, 2, 249-254.

Seamon, J. G. Imagery codes and human information retrieval. Journal of Experimental Psychology, 1972, 96, 468-470.

Senmon, J. G., \& Gazzaniga, M. S. Coding strategies and cerebral laterality effects. Cognitive Psychology, 1973, 5, 249 256.

Sergent, J. About face: Left hemisphere involvement in processing physiognomies. Journal of Experimental Psychology: Human Perception and Performance, 1982, 8, 1-14.
SternberG, S. High-speed scanning in human memory. Science, $1966,153,652-654$

\section{NOTE}

1. Other researchers have found support for parallel processing, when stimuli are somehow organized. For example, Rosenbaum (1974) used digits for memory sets and varied whether the digits followed a rule or not (e.g., multiple of two $\ldots 2,4,6,8$ as a memory set vs. $1,5,6,8)$. DeRosa and Tkacz (1976) presented line drawings that either could or could not be a consecutive sequence (e.g., sequential frames of a golf swing). Both investigations found little or no increase in RT as M increased when the stimuli followed some logical rule or progression.

(Received for publication May 5, 1982; revision accepted October 26, 1982.) 